\title{
A Novel Method for Automated Fractionation Detection in Ventricular Tachycardia
}

\author{
D Gupta ${ }^{1}$, H Hashemi ${ }^{1}$, S Akl ${ }^{1}$, D Redfearn ${ }^{2}$ \\ ${ }^{1}$ Queen's University, Kingston, Canada \\ ${ }^{2}$ Kingston General Hospital, Kingston, Canada
}

\begin{abstract}
Catheter ablation therapy has become a key intervention in treatment of recurrent Ventricular Tachycardia (VT). We propose an automated fractionation detection method for VT patients which can potentially increase the accuracy and success rate of ablation therapy. A train of pacing with three different timings, close to ventricular effective refractory period (VERP), was introduced from right ventricle $(R V)$ apex; surface and intracardiac activations were recorded from different sites of the RV in 10 patients (5 tests, and 5 control). Data was processed with Teager-Kasers Energy Operator for peak detection. Features (including latency, electrogram duration, and deflections) were automatically extracted and used to detect fractionation. Performance was evaluated by comparing the results with a control cohort. Test patients showed a significantly larger mean and standard deviation for all three pace timings, and for all features $(p<0.05)$. All patients showed a significant increase in latency as pacing approached VERP $(p<0.05)$. We showed that this process can be automated, and that the data obtained is correlative with arrhythmia. Furthermore, we showed that this data might be useful in isolating arrhythmogenic tissue for better targeted ablation.
\end{abstract}

\section{Introduction}

Ventricular tachycardia (VT) occurs in patients with structural heart disease and can be associated with an increased risk of sudden death [1]. Conventional techniques such as Implantable Cardioverter-Defibrillators (ICDs) are effective treatments for cardiomyopathy, and have been shown to reduce cardiovascular death and total mortality in patients with reduced ejection fraction after Myocardial Infarction [2]. However, ICDs play a limited role in that they only terminate VTs, not prevent them. Furthermore, ICD shocks can be painful for the patient, they increase mortality, and worsen quality of life [3].

Catheter ablation therapy has progressed significantly over the past few years and is now an important option to control recurrent VTs [1]. Catheter ablation can prevent recurrent VT without the need for long-term antiarrhythmic therapy, and reduce the need for ICD shocks.

Current ablation techniques focus on isolating scar tissue by targeting reentry circuit isthmuses and their exits [4]. To accomplish this, the area of the scar must first be defined with the assistance of an electroanatomical system. Substrate mapping can be performed using voltage, pace mapping, and registration of electrogram features suggestive of abnormal myocardium. Though substrate mapping alone can be used to guide the ablation, combining it with short episodes of activation, and entrainment mapping to isolate fractionation increases the likelihood of successful ablation [4]. However, current fractionation mapping methods used to isolate the ablation targets in VT patients are done manually, and are therefore time consuming. They also have a limited success rate, with an approximately $50 \%$ recurrence rate within 2 years [5].

We propose an automated fractionation detection method for VT patients which can potentially increase the accuracy and success rate of the ablation therapy. Performance is evaluated by comparing the results between the test (patients with VT) and control cohort (normal heart and absence of VT).

\section{Materials}

Intracardiac bipolar electrogram (sampling rate $2034.5 \mathrm{~Hz}$ ) segments were recorded using the impedance based 3D navigation system (EnSite ${ }^{\mathrm{TM}}$ Velocity $^{\mathrm{TM}}$; St. Jude Medical, MN). A pacing catheter (Inquiry ${ }^{\mathrm{TM}}$, St. Jude Medical) was used for pacing and a multipolar circular catheter (Reflexion $\mathrm{HD}^{\mathrm{TM}}$, St. Jude Medical hoitherto called the roving catheter) for recording the electrograms. Data was obtained from five test patients with ischemic cardiomyopathy and recurrent VT requiring treatment

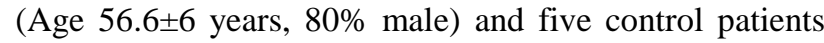
with demonstrated normal left ventricle undergoing left sided ablation for atrial fibrillation (Mean age 53.2 \pm 3.3 
years, $60 \%$ male). A total of 1350 segments, of approximately 30 seconds in duration, were collected.

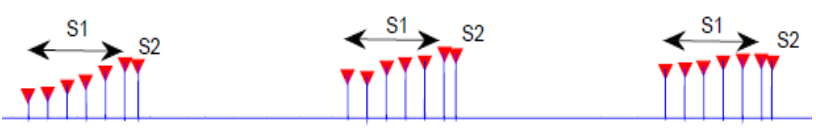

Figure 1. A sample of pacing train with three different timings.

\section{Methods}

Ventricular effective refractory period (VERP) was determined at the right ventricular apex (RVA) using a drive train of $600 \mathrm{~ms}$ (S1) and decreasing extrastimuli (S2) to non-capture. Signals were recorded from the left ventricle (LV) of patients using the roving catheter positioned at various locations guided by the mapping system to ensure complete endocardial coverage. Data were collected during pacing trains from the RVA of 600/(VERP+150), 600/(VERP+100) and 600/(VERP+50) for each data collection site. The S1 consisted of six beats based on the physiological constraints of the patient and technician oversight. An example of a standard pacing train can be seen in Figure 1. The first three pacing spikes are S1 interval and the last pacing spike is the extrastumli (S2).

The data analysis for fractionation detection can be divided into three main processing steps: preprocessing, ventricular peak detection, and feature extraction. Preprocessing consisted of a Discrete Wavelet Transformation based approach [6] to remove baseline wander and low-frequency noise; a Butterworth low pass filter $(300 \mathrm{~Hz})$ to remove high frequency disturbances and lastly, Teagers - Kaiser Energy operator to simplify peak detection (Figure 2). All data analysis was done in Matlab (Mathworks Inc).

\subsection{Teager - Kaiser Energy Operator}

Teager-Kaiser Energy operator (TK) is a nonlinear quadratic operator proposed by Teager [7] to measure the real physical energy of a system $\left(E_{n}\right)$ :

$$
E_{n}=E[x(n)]=x^{2}{ }_{n}-\left(x_{n+1}\right) *\left(x_{n-1}\right)
$$

where $\mathrm{x}(\mathrm{n})$ is a discrete time signal and $\mathrm{n}$ is the discrete time index. This operator is capable of tracking

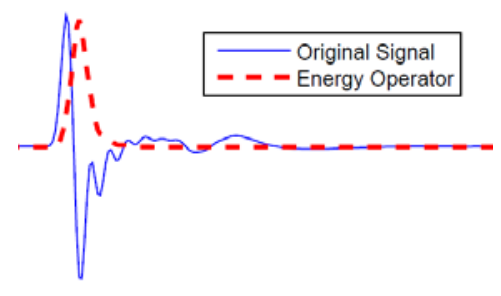

Figure 2. Original (solid) and processed (dotted) signals.

instantaneously-varying special patterns making it ideal for isolating and emphasizing peaks in intracardiac recordings.

\subsection{Peak detection}

Peaks isolated were filtered if their amplitude was too low, or if they were of a distance of less than $250 \mathrm{~ms}$ with respect to their neighbour (VERP is rarely less than $200 \mathrm{~ms}$ [8]). Responses on the surface ECG were monitored to isolate and discard possible anomalies in the data resulting in a non-capture; such as sinus beats and ectopy as shown in Figure 3.

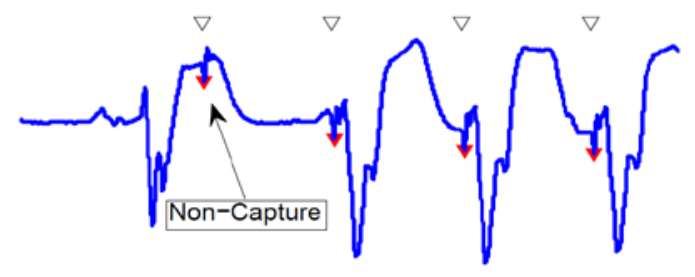

Figure 3. Dark triangles show identified activation on EGM channel, while the clear triangles show the activation from the pacing catheter. Figure shows a noncaptured signal due to a sinus beat before the pace. The marked location would be filtered for being an abnormal train.

\subsection{Feature extraction}

Fractionation in our study was defined by three features: latency (conduction time (CT) normalized to the drive train), local bipolar electrogram (EGM) duration, and number of deflections within the EGM duration window. CT refers to the time between a pacing artifact and the start of a local EGM activation at the target location, this is normalized by subtracting the CT during the drive train at that location during S2 to provide a measure of latency defined at the delay between delivery of the pacing stimulus and onset of local potential. EGM duration refers to the duration of the local activation from start to end; and deflections is the total number of peaks found in the activation. An example of all three can be found in Figure 4. 


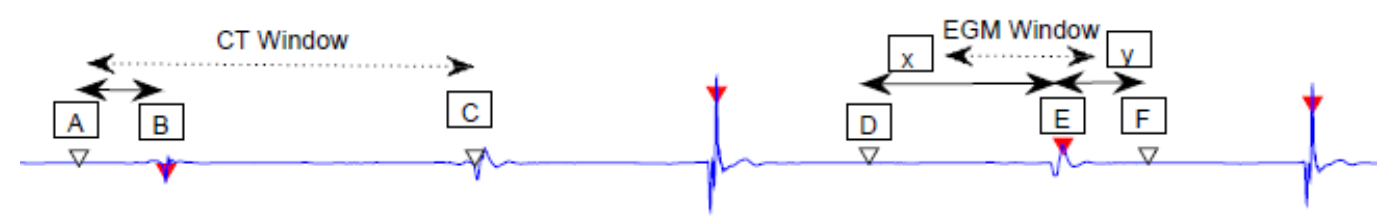

Figure 5. Pacing spikes are shown by the clear triangles, while response spikes are shown by the dark triangles. Sequence on the left shows the steps involved in determining CT. Window in which the response spike was identified was defined by the current pace, and the next pace in the train (A-B, shown by dotted line on left). Within this window the largest peaks are found to isolate response spikes. Sequence on the right shows the steps involved in determining the EGM duration. The EGM window ( $x-y$, shown by the dotted line on the right) is defined by the half way point between the pace and response spike ( $\mathrm{D}$ and $\mathrm{E}$ respectively, the location of which is identified when obtaining $\mathrm{CT}$ ), and the half way point between the response spike and the next pace (E and F respectively).

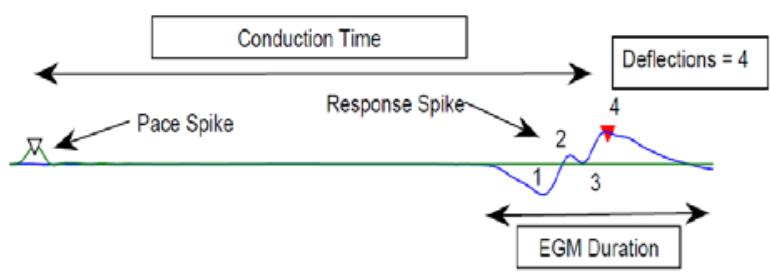

Figure 4. The three features used to represent fractionation: CT, EGM duration, and deflections (all pertinent peaks have been numbered for a sum of 8 for this EGM). Pace spike was taken from the pacing catheter while response spike was taken from the roving catheter.

After pre-processing, these three features were extracted from each recording. CT was identified by linking pacing spikes in the pacing catheter, to response spikes in the roving catheter. Response spikes were identified by isolating the largest signal peak in the roving catheter (point ' $B$ ' in Figure 5) that occurred after a pace (point ' $A$ ' in Figure 5), and before the next pace in the train (point ' $\mathrm{C}$ ' in Figure 5).

\section{4. Statistical analysis}

Data were inspected for normal distribution. Comparison between groups was performed with Mann_ Whitney U test; within patient analysis with KruskallWallis. Normal values were obtained by use of a trimmed mean plus two times the standard deviation.

\section{4. $\quad$ Results and discussion}

The three previously defined features were extracted from all test and control patients. An average of $174 \pm 113$ locations per test patient and $89 \pm 29$ per control patient were obtained. The difference explained by the much larger size of the test ventricles. Figure 6 shows the distribution of data from test and control. The majority of data from both groups lies within the normal range yet, as anticipated, the test patients demonstrate a significantly greater proportion of data outside the normal range and this is exposed with decreasing coupling interval of S2.

Control patients were employed to determine normal responses and data spread, this derivation cohort provide the normal values observed in Figure 6. EGM duration did increase slightly in controls but here was no change in fractionation and only a trend toward increased latency at this coupling interval. This is consistent with prior physiological observation [9] where significant changes only occur at much tighter coupling intervals. However there was a significant change in latency observed in test subjects as expected. There was also a significant difference in latency between both groups at each S2. These data demonstrate the practical utility of the automated process to geographically isolate areas with abnormal responses from areas falling within the normal range.

Table 1 shows the percentage of data that were retained in the test dataset after removal of normal range data. The results show that latency as a function of abnormal behaviour appears to change little with decreasing S2; however it is unclear if individual data points remain stable or if there is change in geographical distribution of latency, this requires further study. More interesting is the response of the EGM characteristics with a reduction in abnormal classification with decreasing S2. This suggests much of this abnormal tissue cannot sustain the faster conduction and physiologically 'block'. This is an important observation as such sites may not be responsible for faster VT and may be targeted 

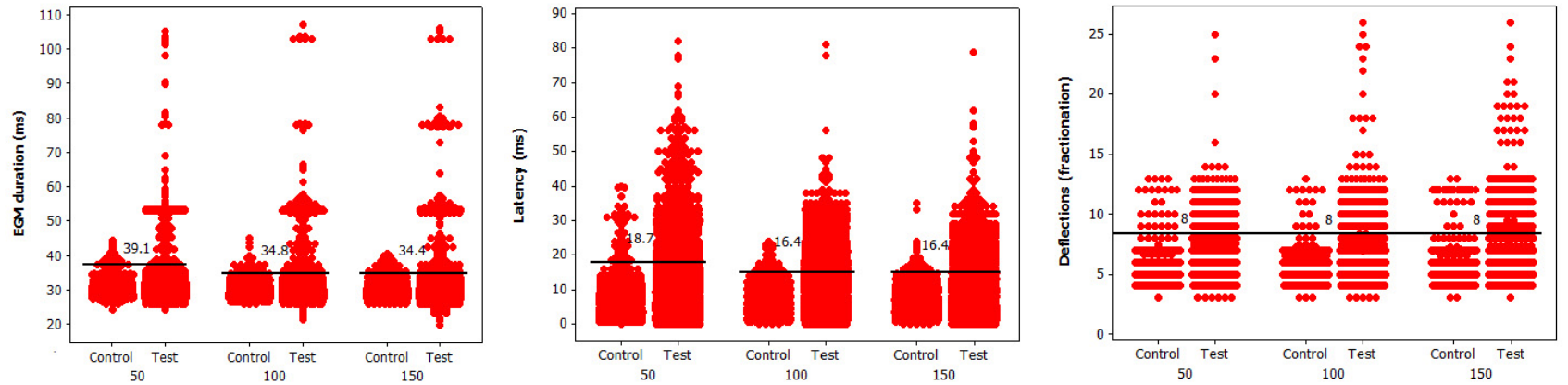

Figure 6. A comparison between Test and Control patients for the features EGM duration, latency and deflections from left to right respectively; for all three pacing intervals. Solid horizontal lines represents the median value for the control patients for specified dataset.

Table 1. Percentages retained after filtering the Test dataset of all values less than the (mean+2*standard deviation) obtained from the control dataset

\begin{tabular}{|c|c|c|c|}
\hline $\begin{array}{l}\text { Extrastimuli } \\
\text { (ERP +) }\end{array}$ & Latency & EGM & Deflections \\
\hline $\mathbf{1 5 0}$ & 34.6 & 32.2 & 16.4 \\
\hline $\mathbf{1 0 0}$ & 30 & 8.7 & 16.8 \\
\hline $\mathbf{5 0}$ & 35.4 & 3.6 & 11.8 \\
\hline
\end{tabular}

as a secondary endpoint. Such physiologic correlations may allow operators to discriminate abnormal substrate much faster and avoid a large amount of possible unnecessary ablation in this cohort.

In this paper, we showed that automated detection of fractionation metrics within VT patients is possible, and that the data obtained is correlative with arrhythmia. Furthermore, we wanted to see if PEFA would be effective at isolating arrhythmogenic tissue for better targeted ablation. The sites defined as abnormal using our controls might be effective in allowing us to do so and can be readily applied to conventional mapping systems.

\section{References}

[1] Koplan BA, Stevenson WG. Ventricular Tachycardia and Sudden Cardiac Death. Mayo Clinic Proceedings. 2009;84(3):289-297.

[2] Moss A.J., Zareba W., Hall W.J., Multicenter Automatic Defibrillator Implantation Trial II Investigators Prophylactic implantation of a defibrillator in patients with myocardial infarction and reduced ejection fraction. $\mathrm{N}$ Engl $\mathrm{J}$ Med. 2004;346:877-883.

[3] Poole JE, Johnson GW, Hellkamp AS, Anderson J, Callans DJ, Raitt MH, Reddy RK, Marchlinski FE, Yee R, Guarnieri T, Talajic M, Wilber DJ, Fishbein DP, Packer DL, Mark DB, Lee KL, Bardy GH. Prognostic importance of defibrillator shocks in patients with heart failure. $N$ Engl $\mathrm{J}$ Med 2008;359:1009-1017.

[4] Soejima K, Suzuki M, Maisel WH, Brunckhorst CB, Delacretaz E, Blier L, Tung S, Khan H, Stevenson WG. Catheter ablation in patients with multiple and unstable ventricular tachycardias after myocardial infarction: short ablation lines guided by reentry circuit isthmuses and sinus rhythm mapping. Circulation 2001;104:664-669.

[5] Jaïs P., Maury P., Khairy P., Elimination of local abnormal ventricular activities: a new end point for substrate modification in patients with scar-related ventricular tachycardia. Circulation. 2012;125:21842196.

[6] Addison PS. Wavelet transforms and the ECG: a review. Physiological Measurement. 2005:25(5):9 R155-R199

[7] Solnik S, Rider P, Steinweg K, DeVita P, Hortobágyi $T$. Teager-Kaiser energy operator signal conditioning improves EMG onset detection. European Journal of Applied Physiology. 2010;110(3):489-498

[8] Linker NJ, Dancy M, Malik M, Jones S, Ward DE. Effect of activation sequence on ventricular refractoriness as determined by extristimuli. Cardiovascular Research. 1990;24(8)659-664

[9] Saumarez RC1, Pytkowski M, Sterlinski M, Bourke JP, Clague JR, Cobbe SM, Connelly DT, Griffith MJ, McKeown PP, McLeod K, Morgan JM, Sadoul N, Chojnowska L, Huang CL, Grace AA. Paced ventricular electrogram fractionation predicts sudden cardiac death in hypertrophic cardiomyopathy. Eur Heart J. 2008 Jul;29(13):1653-61. 